\title{
Cultivo de vainilla en el sur de Florida ${ }^{1}$
}

\section{Alan Chambers, Pamela Moon, Vovener de Verlands Edmond, y Elias Bassil; traducción por Dayana Valdes ${ }^{2}$}

\section{Nombre científico: Vanilla planifolia}

Nombres comunes: orquídea de vainilla

Familia: Orchidaceae

Origen: Mesoamérica

Distribución: Global de latitudes $27^{\circ} \mathrm{N}$ a $27^{\circ} \mathrm{S}$.

\section{Resumen}

El extracto de vainilla es popular en el mundo entero como un ingrediente usado en helados y otros postres. La fuente botánica del extracto de vainilla es principalmente los frijoles curados de Vanilla planifolia. El Estados Unidos es el mayor importador mundial de vainilla judías, pero la producción nacional es mínima. El sur de la Florida tiene un clima de crecimiento favorable para el cultivo de vainilla. Este documento incluye información relevante para los productores interesados en establecer un cultivo de vainilla.

\section{Historia}

Las semillas encapsuladas de vainilla (comúnmente llamados frijoles) siempre han sido apreciadas por su aroma y sabor distinto. La especie comercial principal es V. planifolia mientras que la especie $V . \times$ tahitensis es cultivada en menor medida. Además de Madagascar, que viene siendo el líder del mundo en producción de vainilla, le siguen los países de Indonesia, Uganda, India, Comores, México y otros que contribuyen significativamente a la producción mundial. Los Estados Unidos es el mayor importador del frijol vainilla el cual se convierte a extracto de vainilla. El extracto de vainilla ahora se usa ampliamente en helados, productos horneados, chocolate, cosméticos y muchos otros productos.

V. planifolia extiende desde su nativa gama de Mesoamérica a través de las islas del Caribe, en Europa y en todo el mundo comenzado a finales de los 1500s. Los climas más fríos dependían de los invernaderos para mantener esta especie tropical. Las vides no se produjeron fuera del rango nativo en ausencia de polinizadores naturales. El desarrollo de métodos de polinización manual en 1837 y 1841 por Charles Morren y Edmund Albius, respectivamente, desbloqueó el potencial de esta especie para la producción comercial fuera de Mesoamérica. Este desarrollo oportuno apoyó la expansión de la producción en las décadas de 1850 y 1860 en respuesta a las limitaciones de producción de México. Hoy en día, los descendientes clonales de las plantas originales se cultivan comercialmente en varios países.

La vainilla se ha cultivado en los Estados Unidos desde antes de principios de 1900 en Puerto Rico, Hawái y Florida. V. planifolia se introdujo desde la Florida en Puerto

1. Este documento, HS1350, es uno de una serie de publicaciones del Horticultural Sciences, Servicio de Extensión Cooperativa de la Florida, Instituto de Alimentos y Ciencias Agrícolas, Universidad de la Florida. (UF/IFAS). Fecha de primera publicación: November 2019. Visite nuestro sitio web EDIS en https://edis.ifas.ufl.edu. The English version of this article is HS1348, Vanilla Cultivation in Southern Florida.

2. Alan Chambers, assistant professor, Horticultural Sciences Department; Pamela Moon, biological scientist III; Vovener de Verlands Edmond, graduate student; and Elias Bassil, assistant professor, Horticultural Sciences Department; UF/IFAS Tropical Research and Education Center, Homestead, FL 33031. Traducción por Dayana Valdes.

The Institute of Food and Agricultural Sciences (IFAS) is an Equal Opportunity Institution authorized to provide research, educational information and other services

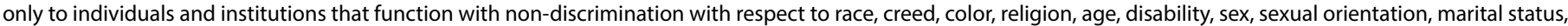

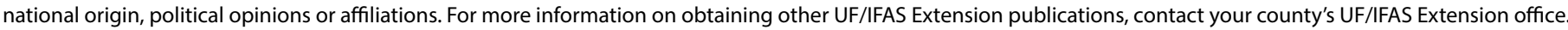
U.S. Department of Agriculture, UF/IFAS Extension Service, University of Florida, IFAS, Florida A \& M University Cooperative Extension Program, and Boards of County Commissioners Cooperating. Nick T. Place, dean for UF/IFAS Extension. 
Rico dos veces antes de 1909. Una industria de la vainilla en Puerto Rico incluía una cooperativa de productores. La estación del USDA en Puerto Rico realizó investigaciones de horticultura, mejoramiento y curado de frijoles en apoyo de la industria de la vainilla. La industria en Puerto Rico creció hasta la década de 1950, cuando disminuyó después de la Segunda Guerra Mundial cuando la industrialización reemplazó a la agricultura. Hawái recibio vainilla como parte de las rutas comerciales antes de 1900. Hawái todavía tiene producción de vainilla, pero sobre todo favorece a los modelos de negocio de turismo. Además, Florida tiene cuatro especies de vainilla nativas ( $V$. barbellata, $V$. dilloniana, V. phaeantha, y V. mexicana) con $V$. planifolia naturalizada (Figura 1). Puerto Rico tiene siete especies que crecen silvestres ( $V$. barbellata, V. dilloniana, V. poitaei, $V$. pompona, V. claviculata, V. pompona y V. planifolia). Las especies nativas de vainilla de Florida están en peligro y no deben ser cosechadas de áreas naturales sin la debida autorización y permiso de las autoridades reguladoras.

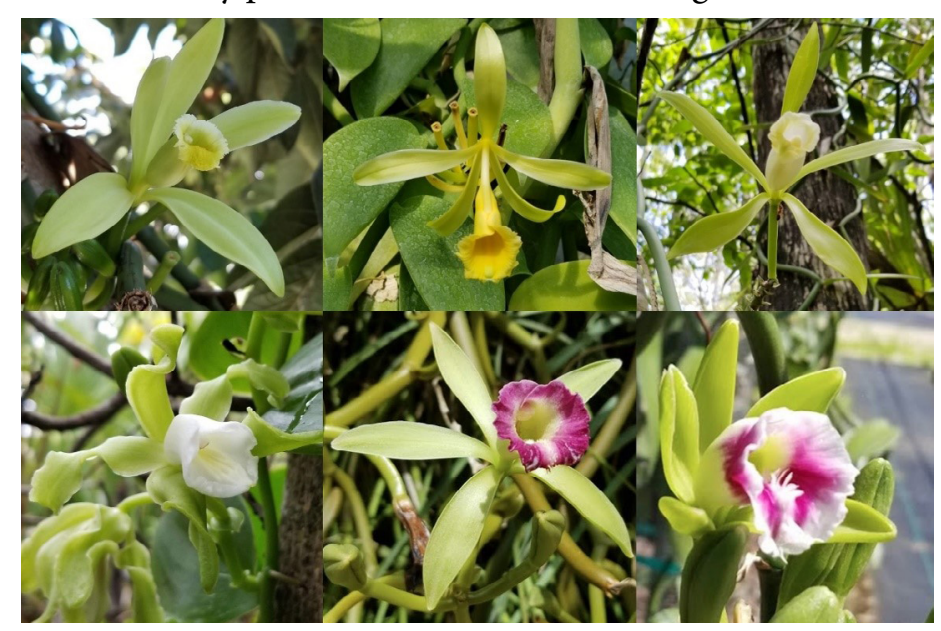

Figura 1. Flores de V. planifolia (arriba a la izquierda), $V$. pompona (arriba en el medio), $V$. phaeantha (arriba a la derecha), $V$. mexicana (abajo a la izquierda), $V$. dilloniana (abajo en el medio) y $V$. barbellata (abajo a la derecha) que crecen en el sur de Florida. Créditos: Alan Chambers, UF/IFAS

\section{Importancia}

El extracto de vainilla es la segunda especia más valiosa (después del azafrán), y es el sabor más popular del mundo. El extracto natural de vainilla proviene predominantemente de los frijoles curados de V. planifolia, que es la especie comercial principal, y en menor medida de $V$. $x$ tahitensis y V. pompona. Los perfiles de aroma y sabor varían para cada especie y para cada entorno de crecimiento. Estas diferencias podrían ser útiles para una variedad de aplicaciones, incluyendo como ingredientes alimentarios, en cosméticos, y para varias otras aplicaciones.

\section{Descripción}

\section{Planta}

V. planifolia es una vid carnosa y perenne con tallos verdes. Las cepas viven por muchos años y algunas especies llegan a crecer hasta $60 \mathrm{~m}$ (200 pies) de altura. El diámetro del tallo aumenta a medida que la planta madura. La vainilla es semi-epífita, lo cual significa que es capaz de enraizarse en el suelo y también crecer en otras plantas sin contacto directo con el suelo.

\section{Hojas}

V. planifolia tiene hojas suculentas de color verde brillante. Las hojas maduras pueden ser de tamaño variable, que van desde $8-25 \mathrm{~cm}$ (3-14 pulgadas) de largo y 2-8 cm (0,75-3 pulgadas) de ancho. Ellas son desde forma lanceoladas hasta ovaladas, con puntas afiladas y pueden sobrevivir unos 3-4 años. Algunos tipos de V. planifolia tienen hojas de formas variadas, y están por lo general crecidas como plantas ornamentales.

\section{Flores y frutas}

Las flores de $V$. planifolia son grandes y fragantes.

Tienen sépalos cerosos color crema verde que forman en inflorescencias axilares. Las flores de V. pompona son amarillas en comparación con $V$. planifolia. Flores pueden alcanzar aproximadamente 6 a $8 \mathrm{~cm}(2,5$ a 3in) de longitud y aproximadamente 5 a $10 \mathrm{~cm}$ ( 2 a 4 pulgadas) de diámetro. Dos de los pétalos son similares en apariencia a los sépalos. El tercer pétalo se modifica en forma de labio. Este labio en forma de pétalo contiene dos polinias (masas de polen) y el estigma, montado en una columna. Una estructura situada entre el estigma y la polinia, llamada rostelo, previene efectivamente la autopolinización.

Las flores se forman en racimos axilares los cuales pueden contener muchas o pocas flores. Ellas aparecen por primera vez 2-3 años después de sembrar un nuevo corte. La vainilla tiende a florecer en vides más grandes cuando el diámetro alcanza 6-13 mm (0.25-0.5 in). Por lo general, una flor, pero a veces hasta tres flores en un grupo pueden abrirse a la vez temprano en la mañana. La floración tiende a ocurrir durante un período de aproximadamente 2 meses, una vez al año, pero cada flor individual de $V$. planifolia dura solo un día. Las flores de algunas otras especies de vainilla pueden permanecer viables por más tiempo que $V$. planifolia.

Después de la polinización, los ovarios se hinchan para producir una semilla larga en forma de cápsula (frijol) que puede alcanzar aproximadamente $20 \mathrm{~cm}$ (8 pulgadas) de 
largo y tomar entre 8-9 meses para madurar. Los granos de vainilla contienen miles de semillas pequeñas, negras, y redondas. Al madurar, el frijol se abre a lo largo de dos costuras longitudinales, exponiendo las semillas y arruinando el frijol para los propósitos comerciales.

\section{Raíz}

La vainilla produce dos tipos de raíces incluyendo raíces aéreas y terrestres. Raíces aéreas generalmente no forman ramas y se forman en el vástago opuestos a las hojas. La función primaria de las raíces aéreas es apoyar la vid que suelen a escalar y son por lo tanto muy eficaz en adherirse a las plantas o estructuras. Raíces terrestres generalmente surgen de la base de la vid, son ramificadas, y poseen pelos de la raíz que se asocia a menudo con micorriza. Al igual que otras raíces terrestres, la función primaria de estas raíces es absorber nutrientes y agua del suelo.

\section{Polinización}

La polinización automática de las flores de $V$. planifolia es rara o inexistente en regiones donde no se producen polinizadores nativos (abejas y, tal vez, colibríes). Aunque no es nativo, el Euglossa dilemma de la abeja orquídea (Figura 2) se ha establecido en el sur de Florida y podría ser un polinizador de las orquídeas de vainilla. De lo contrario, nuestras observaciones en áreas naturales muestran que las especies nativas de vainilla establecerán vainas en ausencia de polinización manual (Figura 3). Una hipótesis es que un polinizador de orquídeas nativas en el sur de Florida también podría polinizar las flores de V. planifolia reduciendo la necesidad de polinización manual.

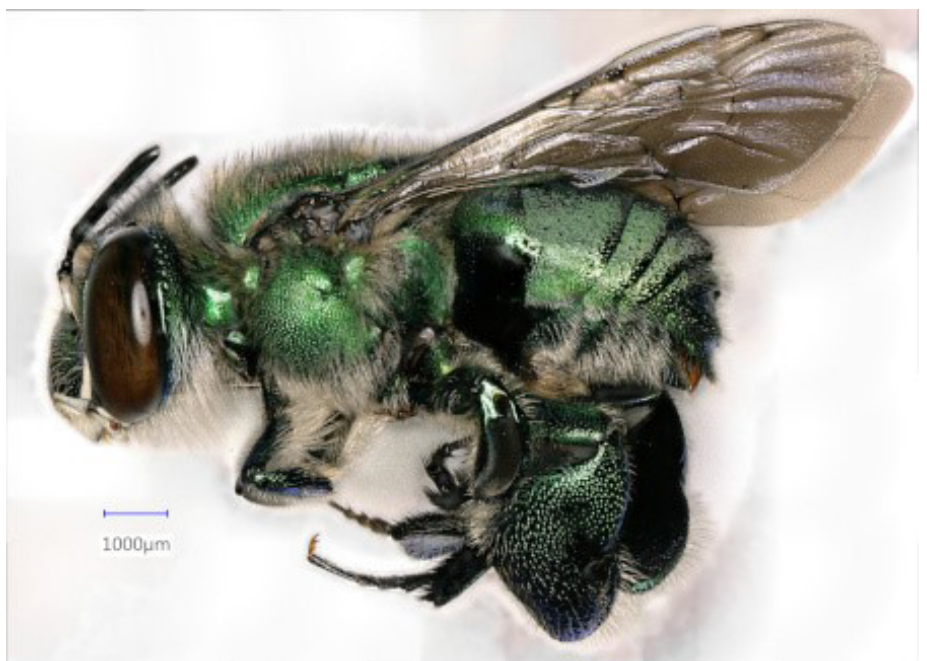

Figura 2. Euglossa dilemma atraído y capturado en el Centro de Investigación y Educación Tropical de la Universidad de Florida. Esta abeja podría ser un polinizador de orquídeas de vainilla. Créditos: Daniel Carrillo, UF/IFAS

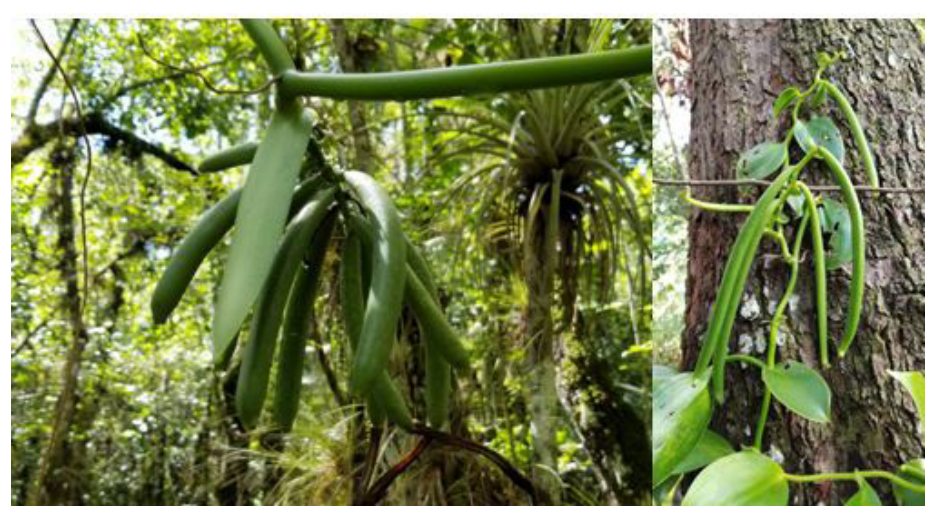

Figura 3. Desarrollo del frijol en ausencia de polinización manual para V. phaeantha (izquierda) y V. mexicana (derecha) en áreas naturales. Créditos: Alan Chambers, UF/IFAS

$V$. planifolia es generalmente auto compatible lo que significa que el polen de una flor puede ser utilizado para fertilizar la misma flor y tendrá lugar a las semillas y el desarrollo del frijol. Según los informes, la polinización es baja $(\sim 1 \%)$ en el rango nativo de México, incluso cuando los polinizadores están presentes. De este modo, la vainilla para producción comercial es muy dependiente de la polinización manual.

Para polinizar una flor de vainilla, él rostelo que separa las polinias y el estigma que tiene que ser pasado por alto. Las polinizaciones deben intentarse por la mañana, generalmente entre las 6 a.m. y el mediodía. La polinización manual se puede lograr con un palillo u otro implemento estrecho. Las secciones de la flor V. planifolia y V. pompona se muestra en la Figura 4 para ayudar en la descripción de la polinización. El pétalo inferior se puede rasgar para exponer las anteras con polen (polinia), rostelo y estigmas. El rostelo se empuja suavemente hacia arriba y lejos de los estigmas hasta que el colgajo de polinia se puede empujar hacia los estigmas y hacer un contacto suave con los estigmas. Si la polinización es exitosa, la flor se mantendrá en la planta, de lo contrario, generalmente se cae en 2-3 días. Los frijoles comenzarán a hincharse y alargarse rápidamente en unas pocas semanas si se polinizan con éxito.

Para los fines comerciales, solo se polinizan las flores en el lado inferior del racimo. Los frijoles producidos a partir de aquí se convertirán en frijoles rectos y tendrán un precio superior. Si la planta es vigorosa, se pueden polinizar un total de 8-12 flores en 10-20 racimos. Alrededor de 10 frijoles por racimo es deseable. 


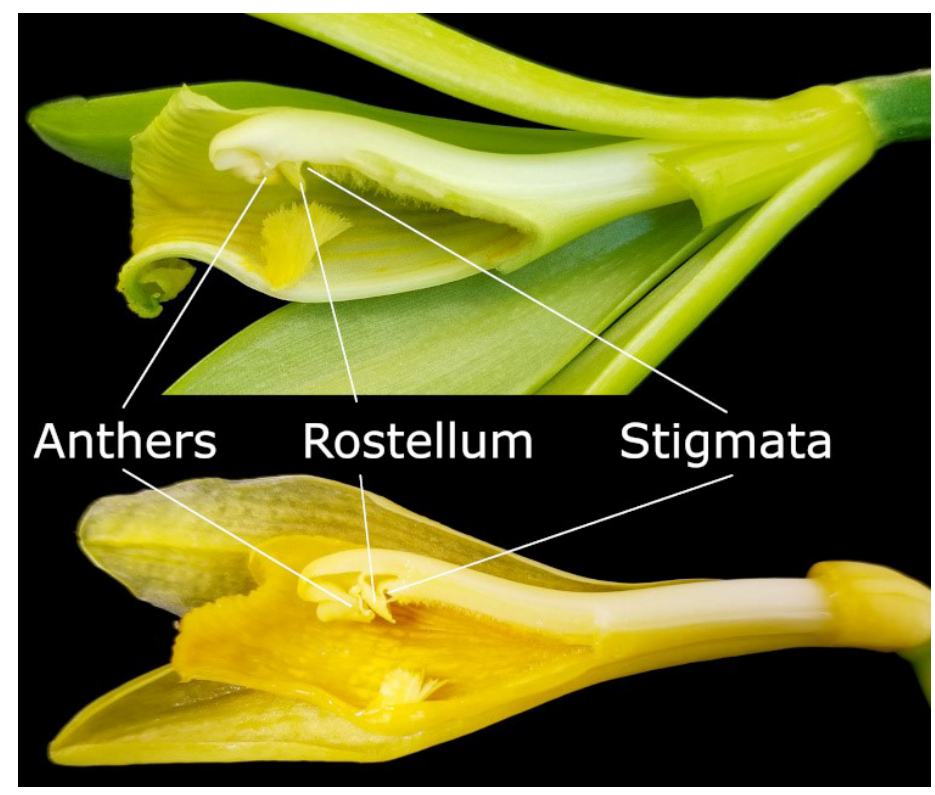

Figura 4. V. planifolia (arriba) y V. pompona (parte inferior) de la flor $s$ con anteras (polinias), rostelo, y estigmas. Los estigmas están protegidos directamente detrás del rostelo.

Créditos: Alan Chambers, UF/IFAS

\section{Variedades}

$V$. planifolia generalmente no se ha beneficiado del fitomejoramiento moderno, por lo que existen pocos cultivares con nombre. Solo se ha patentado un cultivar único, 'Handa'. Esta variedad fue desarrollada por investigadores de la Isla de la Reunión, y se desconoce la disponibilidad futura de este material. De lo contrario, se han caracterizado algunos tipos distinguibles de V. planifolia. Estos incluyen los tipos 'Mansa' originarios de México y que se cultivan comúnmente para la producción comercial. También hay dos tipos de V. planifolia variegada generalmente disponibles por internet y cultivados solo para fines ornamentales.

$V . \times$ tahitensis es el segundo tipo de vainilla cultivado a escala comercial. Nuestra comprensión actual es que $V . \times$ tahitensis es principalmente $V$. planifolia genéticamente con un poco de $V$. odorata (otra especie de vainilla) en su ascendencia. La " $x$ " denota que este es un híbrido y no una especie separada. La contribución genética de $V$. odorata a $V . \times$ tahitensis parece ser menor, pero tiene un gran impacto en la división y el aroma del frijol. El aumento de las notas de sabor a anís de este tipo es popular en los postres franceses. Esquejes que dicen ser $V . \times$ tahitensis se pueden comprar por internet, pero nuestra investigación ha encontrado que muchos vendedores en línea están vendiendo, esperamos que sea por desconocimiento, $V$. planifolia como si fuese $V . \times$ tahitensis. La mayoría de las fuentes internacionales de $V . \times$ tahitensis verificadas no están dispuestas a compartir su material. Se están conduciendo investigaciones para verificar y distribuir $V . \times$ tahitensis verificada a los productores interesados.

También hay riesgos asociados con la compra de plantas en stock que tienen cualidades no deseadas, incluidos los tipos estériles, de bajo rendimiento o de baja calidad. Muchos de estos producirán la vid y las flores clásicamente hermosas, pero no son óptimas para el cultivo comercial. Desafortunadamente, las fuentes de estos tipos son difíciles de rastrear debido al tiempo que lleva obtener una vid madura y floreciente. Actualmente se están realizando investigaciones para identificar los tipos que pueden producir consistentemente frijoles de calidad comercial para reducir el riesgo para los productores.

V. pompona se cultiva comúnmente en el sur de Florida por aficionados y también se confunde comúnmente con $V$. planifolia. V. pompona es vigorosa, pero según se informa produce un extracto de menor calidad. Las flores de $V$. pompona son amarillas en comparación con el color verde crema de las flores de V. planifolia.

\section{Condiciones ambientales}

El sur de Florida, con su clima cálido y húmedo, es adecuado para el cultivo de vainilla. La vainilla es sensible a las bajas temperaturas, incluida la congelación. Por lo tanto, se debe seleccionar un lugar con riesgo reducido de eventos de congelación para el cultivo de vainilla. Las precipitaciones óptimas para vainilla se han informado alrededor de 170 a $280 \mathrm{~cm}$ por año (67 a 110 pulgadas / año). Vainilla requiere alrededor de los dos meses de la estación seca para iniciar floración. Las condiciones de humedad excesiva durante la maduración de la cápsula pueden conducir a grano de la putrefacción. El riego suplementario puede ser útil para establecer nuevos esquejes y potencialmente para la protección contra heladas. La Figura 5 muestra la precipitación y las temperaturas mensuales promedio registradas por la estación meteorológica FAWN en el Centro de Investigación y Educación Tropical de la Universidad de Florida.

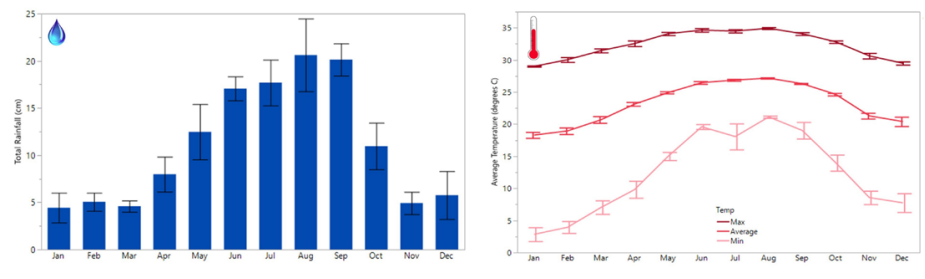

Figura 5. Promedio mensual de precipitaciones y temperaturas máximas, medias y mínimas para Homestead, FL. Los datos son promedios de 10 años entre 2006 y 2016. 


\section{Propagación}

La vainilla se propaga principalmente por esquejes. Es importante dejar que los sitios de corte sanen antes de plantar dejando los recortes de pescado a temperatura ambiente con poca luz durante 1-2 días. Todos los demás factores son iguales, cuanto más largo sea el corte, más rápido se establecerá la vid y comenzará a florecer. Los esquejes de 30 cm (12 pulgadas) de largo generalmente requerirán de 3 a 4 años para florecer, mientras que los esquejes de un metro de largo deben florecer en 2-3 años.

Los esquejes se pueden colocar directamente sobre el sustrato de crecimiento (generalmente mantillo) tan pronto que los sitios de corte se hayan curado. Nebulización ayudará a que las vides retengan las hojas y apoya el desarrollo rápido de nuevas raíces y crecimiento. El extremo apical de los cortes más largos se puede atar a un poste de soporte con solo los 1-2 nodos distales en contacto con el sustrato. Las hojas deben retirarse de los nodos que están en contacto con el sustrato en crecimiento.

Cultivo de tejidos de las plantas V. planifolia están disponibles en algunas compañías. Las plantas crecidas de cultivo de tejidos son atractivas como fuente limpia de material de partida, pero tardarán de 3 a 4 años en madurar y florecer. Las plantas de cultivo de tejidos recibidas en recipientes cerrados deberán endurecerse en una mezcla sin suelo bajo domos de humedad y luz indirecta brillante durante algunas semanas o meses antes de que puedan sobrevivir en un ambiente con menor humedad y aire que fluye libremente. La nebulización ligera y regular aumentará la supervivencia de las plantas jóvenes de cultivo de tejidos.

La vainilla no se propaga comúnmente por semillas debido a los desafíos de germinación. La cubierta de la semilla es lignificada y espesa lo cual impide la germinación oportuna y tomar significativamente más tiempo para crecer en plantas maduras que esquejes. Además, la germinación de semillas probablemente depende de asociaciones con hongos u otros microorganismos. Tales restricciones han apoyado el uso de esquejes como método de propagación primario.

\section{Métodos de producción}

Las vides de vainilla requieren espalderas para maximizar la producción. Se utilizan dos métodos principales de producción. Uno utiliza árboles "tutor" para dar sombra y una estructura adecuada en la que las vides pueden escalar. Árboles de apoyo pueden ser seleccionados en base a la resistencia en un lugar, dada la disponibilidad, consideraciones y co-cultivo. Este tipo de cultivo puede ser menos costoso en algunas áreas, y también reduce naturalmente el riesgo de muerte de la vid por Fusarium al aumentar la distancia entre las plantas. Los productores en el sur de Florida deberían considerar la vainilla como un cultivo secundario en los árboles frutales existentes. La Figura 6 muestra $V$. pompona creciendo en un árbol de aguacate. Cualquier insumo agrícola deberá ser compatible con ambas especies bajo el modelo de cultivos intercalados.

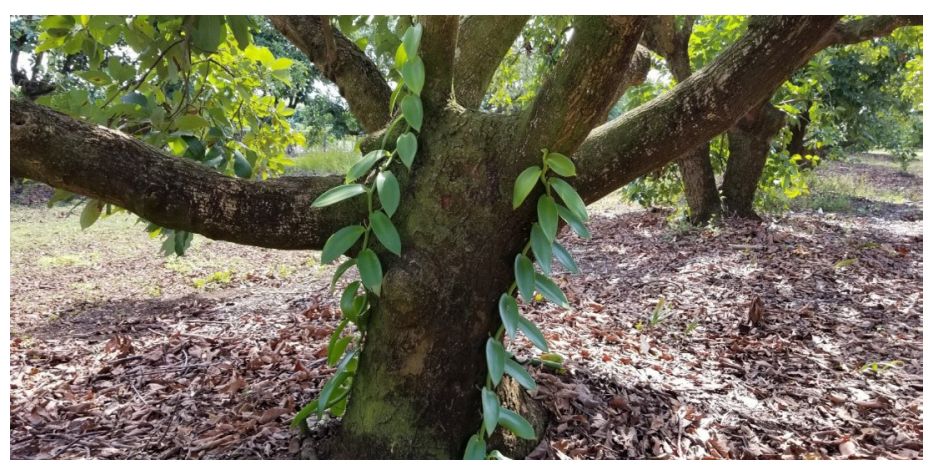

Figura 6. V. pompona que crece en un árbol de aguacate. Las vides se recibieron como esquejes de $\sim 1$ metro de largo. El crecimiento es después de un año sin riego suplementario.

Créditos: Alan Chambers, UF/IFAS

Un cultivo más intensivo bajo estructuras de sombra puede aumentar los rendimientos. Este sistema requiere más inversión inicial para infraestructura, pero permite una mayor densidad de siembra y potencial de rendimiento. Sistemas de apoyo enrejados varían en gran medida, pero generalmente son hechas de madera verticales o soportes de hormigón con alambre que corre entre ellos. Los soportes varían en altura, pero generalmente no tienen más de $2 \mathrm{~m}$ (6 pies) de altura para facilitar la polinización una vez que las vides maduran. El sistema de postes y alambres permite un mayor control sobre el espacio de la vid en comparación con los árboles tutor. Las enredaderas deberán mantenerse en $15-20 \mathrm{~cm}$ (6-8 pulgadas) de sustrato de mantillo.

\section{Espaciado y plantación}

Se estima que a menudo se usan 1,000 plantas por acre para establecer una vainilla comercial. Espaciado recomendaciones son para plantar los vides $1-3 \mathrm{~m}$ ( 3 a 10 pies) de separación, con y 2,5 a $3 \mathrm{~m}$ (8 a 10 pies) entre filas.

\section{Suelos}

Vainilla se puede cultivar en una amplia gama de tipos de suelo, pero prospera en suelos ligeros con abundancia de material orgánico. Para el método del árbol tutor, una ligera pendiente puede ser beneficiosa para reducir la incidencia del agua estancada y la incidencia de enfermedades. Los suelos secos requerirán riego adicional para mantener la humedad adecuada del suelo. Bajo sistemas de cultivo más 
intensivos, la humedad del suelo se puede regular manejando la altura de los lechos de mantillo elevados.

El mantillo es un sustrato popular para el cultivo de vainilla. El tipo particular de mantillo no es tan importante como su capacidad para proporcionar una liberación lenta de nutrientes y retener un nivel óptimo de humedad para las raíces. Algunas áreas dependen de cáscaras de coco envejecidas para cubrir las enredaderas. Será necesario volver a aplicar mantillo cada 6 a 12 meses para reponer la fuente de nutrientes y suprimir las hierbas malas. Se puede aplicar mucho directamente sobre el suelo residente sin la necesidad de incorporarlo al suelo.

\section{Poda y Entrenamiento}

Las vinas están capacitadas para facilitar la polinización manual y la recolección en un proceso llamado bucle. Vides deben pasar alrededor de apoyo enrejados o ramas en forma de bucle según van creciendo. La colocación de enredaderas en el suelo estimulará la formación de raíces terrestres, especialmente si están cubiertas de mantillo, lo que dará lugar a enredaderas más fuertes.

Las vides en bucle y sanas de plantas maduras se pueden inclinar (quitar el ápice) para inducir la floración. Las puntas de las venas se cortan unos $15 \mathrm{~cm}$ (6 pulgadas) desde el extremo (por encima de la línea de tierra) justo antes de la estación seca. Estas vides serán preparadas para florecer en los próximos meses.

\section{Irrigación}

Los ciclos naturales de lluvia de Florida son favorables para la producción de vainilla. La temporada de lluvias, junto con las altas temperaturas del verano estimulan un crecimiento rápido. La estación más seca induce un período de descanso necesario antes de la floración. Como resultado, las plantas de vainilla establecidas a veces pueden no requerir riego suplementario, excepto durante períodos extremadamente secos. Las plantas de vainilla son tolerantes a cortos períodos de desecación. El riego es más crítico para la producción comercial que en el paisaje doméstico.

\section{Fertilización}

El cultivo de vainilla se basa en la liberación lenta de nutrientes del material orgánico en descomposición. Se puede aplicar nutrición foliar suplementaria, pero todavía faltan pruebas validadas científicamente para justificar este aporte adicional.

\section{Plagas}

Las plagas de insectos generalmente no suelen causar daños graves. Los caracoles y las babosas, sin embargo, a veces pueden ser problemáticos si no se controlan. Hemos notado la alimentación de las larvas en las plantas jóvenes, pero las retiramos manualmente.

\section{Enfermedades}

Una limitación importante para la producción de vainilla en muchas regiones es la enfermedad de pudrición de raíz y tallo causada por Fusarium oxysporum. Fusarium es un hongo ubicuo en el suelo que causa podredumbre en muchas especies. Un tipo especializado (Fusarium oxysporum f. sp. radices-vanillae) causa podredumbre en la vainilla en todas las áreas productoras principales al penetrar las raíces y propagarse por toda la planta. Los síntomas típicos incluyen dorado y marchitamiento, que eventualmente conducen a la muerte de las vides. La enfermedad puede controlarse parcialmente mediante buenas prácticas hortícolas, incluida la prevención del anegamiento excesivo. Los fungicidas y los agentes de biocontrol pueden tener algún beneficio bajo ciertas condiciones. Otras posibles enfermedades fúngicas incluyen antracnosis y moho.

\section{Cosecha y curado tradicional}

Los frijoles comienzan a amarillear en el extremo de la flor cuando están completamente maduros. Debe tenerse cuidado de no cosechar los granos antes de tiempo ya que esto repercute negativamente en la calidad del extracto resultante. Los frijoles $V$. planifolia que se dejan en la vid durante demasiado tiempo tienen una mayor propensión a partirse o moldearse, lo que elimina su valor económico. La excepción a esto es $V$. $\times$ tahitensis que no muestra el rasgo de división del frijol y se puede dejar en la vid hasta que esté completamente marrón. Los frijoles individuales deben retirarse cuidadosamente del racimo para evitar dañar los frijoles.

Los granos de vainilla deben ser curados con el fin de desarrollar el aroma y sabor característica de vainilla. Los resultados de curado varían mucho según la ubicación y están fuertemente influenciados por el entorno de crecimiento, la genética de las plantas y la madurez de los frijoles. El curado de los granos de vainilla sigue siendo algo así como un arte con muchas variaciones que se utilizan en diferentes partes del mundo. En general, el curado incluye algunos pasos importantes: clasificación, muerte, sudoración, secado gradual y acondicionamiento. 


\section{Clasificación}

Los frijoles se clasifican en clases que incluyen frijoles de 16 cm y más largos, frijoles de 12-16 cm y frijoles pequeños / divididos / rechazados. Clasificar los frijoles por tamaño es importante para el paso de curado por calor.

\section{Matar con Calor}

El calor se aplica a una temperatura que es lo suficientemente alta como para matar las células de la planta, pero no tan alta que las enzimas requeridas para producir los deseables vainilla sabores serían destruidos. Se requiere matar el agua a $63^{\circ} \mathrm{C}-65^{\circ} \mathrm{C}$ para detener el desarrollo celular y comenzar el proceso de curado. Los frijoles largos $(>16 \mathrm{~cm})$ deben calentarse durante 3 minutos, con frijoles de $12-16 \mathrm{~cm}$ calentados durante 2.5 minutos. Los frijoles más pequeños se pueden matar con agua durante 2 minutos, según las necesidades del producto final. Después de matar el calor, los frijoles se empaquetan en bolsas de plástico para sudar.

\section{Sudoración}

La sudoración es el proceso de mantener elevadas las temperaturas del frijol por encima de $45^{\circ} \mathrm{C}$ durante $24-48$ horas después de matar el calor. Esto se puede hacer usando cajas aisladas con control de temperatura o colocando botellas con agua caliente $\left(\sim 60^{\circ} \mathrm{C}\right)$ en el lado del recipiente aislado. Los frijoles deben ser de color marrón oscuro después de matar el agua y sudar.

\section{Secado gradual}

El secado lento de los frijoles hasta un contenido de humedad final $<25 \%$ es importante para el desarrollo del sabor y el control del crecimiento microbiano. Esto se logra tradicionalmente durante 12-15 días retirando los frijoles de sus bolsas de plástico, exponiéndolos a la luz solar directa durante unas horas cada día y volviendo a empacarlos en bolsas de plástico al final del tratamiento solar. Este proceso podría imitarse utilizando espacios que tengan temperatura, humedad y control de flujo de aire. Los frijoles se colocan en bandejas abiertas para continuar muriendo durante $\sim 70$ días. Los frijoles se clasifican por calidad al final de la etapa de secado. Cualquier frijol mohoso debe descartarse a medida que se detecta.

\section{Acondicionamiento}

El acondicionamiento generalmente toma otros 1-2 meses. Los frijoles empaquetados deben acondicionarse en cajas forradas con papel impermeable a la grasa y al aceite (por ejemplo, papel encerado, papel pergamino, papel a prueba de grasa). No se debe permitir que los frijoles se sequen completamente durante el acondicionamiento.

\section{Hacer tu propio extracto de vainilla}

Solo el extracto de dos tipos de vainilla, $V$. planifolia y $V$. $\times$ tahitensis, puede venderse como "extracto de vainilla" bajo el Código de Regulaciones Federales (Código de Regulaciones Federales Título 21 CFR169.175 y CFR169.3). La regulación dicta que el solvente para el extracto de vainilla sea no menos del $35 \%$ de alcohol etílico en volumen con 13.35 onzas de vainilla con un contenido de humedad del $25 \%$ por galón de solvente. Los productores más pequeños pueden desear considerar la formación de empresas cooperativas para mejorar los procesos de curado y almacenamiento, y para ayudar a asegurar la uniformidad y consistencia de los curados vainilla frijoles.

\section{Economía básica}

Muchos productores potenciales desearían estimaciones básicas para los insumos clave al considerar el inicio de una nueva vainilla. Los datos disponibles provienen de otros entornos de cultivo y prácticas de producción que no necesariamente reflejarán los de los productores del sur de Florida. Aun así, las estimaciones aproximadas pueden ser útiles cuando se considera una nueva operación de crecimiento. Las siguientes respuestas a preguntas comunes se proporcionan solo con fines de estimación, y los resultados reales deberán determinarse empíricamente.

\section{Respuestas a preguntas comunes}

P: ¿Cuántos años hasta que una vid de vainilla produzca flores y frijoles?

R: Los esquejes de un metro de largo se producirán en 2-3 años, y los esquejes más pequeños y las plantas de cultivo de tejidos demorarán de 3 a 4 años.

P: ¿Cuántas veces florece V. planifolia por año y cuándo ocurre?

R: La vainilla florece una vez al año, generalmente en abril-mayo en el sur de Florida.

P: ¿Cuántos meses tarda un frijol en madurar?

R: Aproximadamente 9 meses después de la polinización. 
P: ¿Cuántos kilogramos de judías verdes puede producir una planta de $V$. planifolia? ¿Cuántos kilogramos de frijoles curados?

R: Una planta de vainilla saludable puede producir $\sim 2$ $\mathrm{kg}$ de judías verdes por planta. El curado es habitualmente de aproximadamente 5: haba $1 \mathrm{~kg}$ verde para frijol curado: 1 o 6 por peso, por lo que cada planta puede producir alrededor de 0.3 a $0.4 \mathrm{~kg}$ curados vainilla frijoles.

P: ¿Cuántos frijoles curados necesito para obtener $1 \mathrm{~kg}$ de frijoles?

R: Se necesitarán 200-400 frijoles curados para hacer $1 \mathrm{~kg}$ de frijoles.

P: ¿Cuántas plantas necesito por acre?

R: Alrededor de 1,000 plantas por acre es una buena estimación, pero esto depende del método de producción con más plantas necesarias para el cultivo de la casa, de color intenso y un menor número total de plantas cuando se utilizan árboles de apoyo.

P: ¿Dónde puedo obtener las plantas de V. planifolia?

R: Las empresas de cultivo de tejidos en Florida y fuera de los EE. UU. (Por ejemplo, Costa Rica) pueden identificarse mediante una búsqueda rápida en Internet. Se debe tener precaución para asegurarse de que estas compañías estén vendiendo $V$. planifolia o $V . \times$ tahitensis si planea vender "extracto de vainilla".

P: ¿Cuánto trabajo se requiere durante la polinización?

R: Una estimación general es que una persona puede polinizar un acre de vides de vainilla. Esto incluye monitorear las plantas todos los días durante la temporada de floración para polinizar las flores recién abiertas.

P: ¿Las flores de vainilla realmente necesitan ser polinizadas la mañana que abren?

R: Sí, las flores de vainilla solo son receptivas a la polinización por un corto tiempo. Las temperaturas pueden afectar la longevidad de las flores, pero la polinización antes del mediodía suele ser óptima.

P: ¿Puedo cultivar híbridos de vainilla?

R: Ciertamente, pero el proceso regulatorio para algunos híbridos no está muy claro. Un productor debe considerar a sus compradores y consumidores al considerar el cultivo de híbridos de vainilla que incorporan especies distintas de $V$. planifolia y $V . \times$ tahitensis. Algunos híbridos serán más robustos y tendrán una mayor resistencia a las enfermedades que el V. planifolia tradicional.

P: ¿Cuántas flores deben polinizarse por racimo?

R: Por lo general, no se debe permitir que se desarrollen más de 10 frijoles por racimo. Más podría sobrecargar el estado nutricional de la vid y conducir a una mala salud de la vid al año siguiente.

P: ¿Cuánto tiempo permanecerán en producción las vides de vainilla?

R: Esto depende de la salud de las vides, enfermedades y otras prácticas culturales. Puede anticipar que las vides se mantendrán productivas durante 3-5 años, pero el ciclado regular con vides nuevas y libres de enfermedades ayudará a su operación a producir frijoles de manera sostenible.

\section{Recursos adicionales}

Bianchessi, P. 2012. Vanilla Handbook. Technical Centre for Agricultural and Rural Cooperation.

\section{Cameron, K. 2012. Vanilla Orchids: Natural History and} Cultivation. Timber Press.

Childers, N. F. 1948. Vanilla Culture in Puerto Rico. US Department of Agriculture, Washington, D.C.

Fouche, J. G., and L. Jouve. 1999. "Vanilla planifolia: History, Botany and Culture in Reunion Island." Agronomie 19: 689-703.

Havkin-Frenkel, D., and F. C. Belanger. 2018. Handbook of Vanilla Science and Technology. Wiley-Blackwell, Singapore.

Odoux, E., and M. Grisoni. 2010. Vanilla. CRC Press. 